\title{
Analysis of spatial light modulation characteristics of $\mathrm{C}_{60}$
}

\author{
Sukhdev Roy a) and C. P. Singh \\ Department of Physics and Computer Science, Dayalbagh Educational Institute (Deemed University), \\ Dayalbagh, Agra 282 005, India \\ K. P. J. Reddy ${ }^{\text {b) }}$ \\ Department of Aerospace Engineering, Indian Institute of Science, Bangalore 560 012, India
}

\begin{abstract}
Spatial light modulation based on nonlinear absorption of light by excited-state molecules in $\mathrm{C}_{60}$-toluene solution is demonstrated. Amplitude modulation of probe laser beam transmissions at 747 and $885 \mathrm{~nm}$, corresponding to the peak absorption of $T_{1}$ and $S_{1}$ states, by the modulation laser beam intensity induced population changes at $532 \mathrm{~nm}$ has been analyzed using the rate equation approach, for various values of the small-signal absorption coefficient. It is shown that for $T_{1}$ and $S_{1}$ states, modulation up to $100 \%$ and $94.5 \%$ can be achieved for a laser beam intensity of 800 $\mathrm{W} / \mathrm{cm}^{2}$ and $1 \mathrm{~kW} / \mathrm{cm}^{2}$ at $532 \mathrm{~nm}$, respectively.
\end{abstract}

Development of optically addressed real-time reusable two-dimensional input-output transducers or spatial light modulators (SLMs) is essential for the exploitation of the high-speed processing capabilities offered by opticalprocessing and computing systems. ${ }^{1}$ In addition to being used as input and output transducers, SLMs can also be used for image amplification, time/space transformation, scratch pad memory, programmable detector masking, and page composition for holographic and three-dimensional (3D) memories. ${ }^{1,2}$ The key element in the development of SLMs is the fast response photosensitive material that is free from photodegradation effects usually encountered in organic dye molecules. ${ }^{2,3}$ Hence, efforts are being made to develop suitable materials. ${ }^{4,5}$ Recently the fullerene $\mathrm{C}_{60}$ molecule has evolved as a potential candidate for the development of many photonic devices. ${ }^{6,7}$ Use of $\mathrm{C}_{60}$ molecules for the development of power limiters for eye and sensor protection, ${ }^{8-16}$ pure optical bistable devices, optical switches and logic gates, ${ }^{13,17,18} 3 \mathrm{D}$ data storage, ${ }^{19}$ and solar cells ${ }^{20}$ has been proposed recently.

One of the important features of the $\mathrm{C}_{60}$ molecule is the exhibition of strong reverse saturable absorption (RSA), where a long-lived triplet state has an absorption cross section greater than the ground-state absorption cross section over the complete visible spectrum. ${ }^{6-20}$ In this letter, we present an analysis for the transmission characteristics of $\mathrm{C}_{60}$ molecules using the rate equation approach and propose a model for the development of molecular SLM using these molecules.

The energy-level diagram of $\mathrm{C}_{60}$ is shown in Fig. 1. The linear absorption spectra of $\mathrm{C}_{60}$-toluene solution for ground state $S_{0}$, singlet state $S_{1}$ and triplet excited state $T_{1}$ indicate that in the visible and near-infrared wavelength region, the absorption of the ground state is much smaller than that of the excited states $S_{1}$ and $T_{1} \cdot{ }^{17}$ Molecules from the ground state $S_{0}$ can be excited by laser radiation at $532 \mathrm{~nm}$ to a

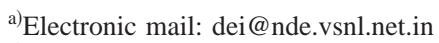

${ }^{b)}$ Electronic mail: laser@aero.iisc.ernet.in vibrational state $S_{v}$ of the first electronic excited state $S_{1}$. The excited molecules rapidly decay in the picosecond time scale to the $S_{1}$ state from where most of them make a transition to the $T_{1}$ state with a relaxation time of $\sim 1 \mathrm{~ns}$. The molecules in $S_{1}$ and $T_{1}$ can be further excited to higherenergy states, but since their lifetimes are extremely short (less than ps), the populations of these higher-energy levels is neglected in our analysis. ${ }^{16,17}$

The light-intensity-induced population changes in different energy levels can be described by rate equations in the operator form,

$$
\frac{d N}{d t}=\hat{O} N,
$$

where operator $\hat{O}$ is defined in terms of the rate constants of different levels as,

$$
\hat{O}=\left[\begin{array}{ccc}
-I_{m} \sigma_{0} & \tau_{S 0}^{-1} & \tau_{T 0}^{-1} \\
I_{m} \sigma_{0} & -\left(\tau_{S 0}^{-1}+\tau_{S T}^{-1}\right) & 0 \\
0 & \tau_{S T}^{-1} & -\tau_{T 0}^{-1}
\end{array}\right],
$$

and the transpose of the population vector $\mathbf{N}$ is given by $\widetilde{N}$ $=\left(N_{1}, N_{2}, N_{3}\right)$.

In this model we have assumed that $N_{i}, i=1-3$, are the population densities; $\sigma_{0}, \sigma_{S}$, and $\sigma_{T}$ are the absorption cross sections of $S_{0}, S_{1}$, and $T_{1}$ states, respectively; $\tau_{S 0}$,

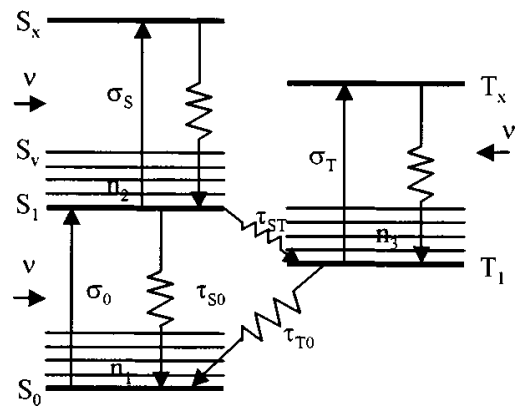

FIG. 1. Energy-level diagram of a $\mathrm{C}_{60}$ molecule. 
TABLE I. Absorption coefficient and transmittance of $\mathrm{C}_{60}$ at different probe wavelengths.

\begin{tabular}{ccc}
\hline \hline$\lambda_{p}(\mathrm{~nm})$ & $\alpha_{p}{ }^{\mathrm{a}}$ & $\left(I_{\text {pout }} / I_{\text {pin }}\right)$ \\
\hline 747 & $N_{3} \sigma_{T p}$ & $\exp \left(-\beta \tau_{S 0} \tau_{T 0} \sigma_{0} I_{m} /\left[\left(\tau_{S 0}+\tau_{S T}\right) X\right)\right] ; \beta=\sigma_{T p} N L$ \\
885 & $N_{2} \sigma_{S p}$ & $\exp \left(-\beta \tau_{S 0} \tau_{S T} \sigma_{0} I_{m} /\left[\left(\tau_{S 0}+\tau_{S T}\right) X\right)\right] ; \beta=\sigma_{S p} N L$ \\
\hline \hline
\end{tabular}

${ }^{a}$ The subscripts $p$ and $m$ signify that the parameters are at the probe wavelength $\lambda_{p}$ and at the modulation wavelength $\lambda_{m}$, respectively. The smallsignal absorption coefficient is $\beta, N$ is the total number of active $\mathrm{C}_{60}$ molecules, and $L$ is the thickness of the medium.

$\tau_{T 0}$, and $\tau_{S T}$ are the relaxation times for nonradiative transitions $S_{1} \rightarrow S_{0}, T_{1} \rightarrow S_{0}$, and $S_{1} \rightarrow T_{1}$, respectively; and $I_{m}$ is the photon density flux of the modulation laser beam (ratio of the intensity $I_{m}^{\prime}$, to the photon energy $h v$ ). Assuming a cw or quasi-cw modulation laser beam with broad light pulses, the light-induced population densities in various levels at steady state are given by

$$
N_{i}=N_{1}\left(\tau_{S 0}^{-1}+\tau_{S T}^{-1}\right)^{-1} I_{m}\left[\begin{array}{c}
\left(\tau_{S 0}^{-1}+\tau_{S T}^{-1}\right) I_{m}^{-1} \\
\sigma_{0} \\
\sigma_{0} \tau_{T 0} \tau_{S T}^{-1}
\end{array}\right],
$$

where, $N_{1}=N / X$ is the population density in $S_{0}, X=1$ $+\sigma_{0} I_{m}\left(\tau_{S 0}^{-1}+\tau_{S T}^{-1}\right)^{-1}\left(1+\tau_{T 0} \tau_{S T}^{-1}\right)$, and $N=N_{1}+N_{2}+N_{3}$ is the total number of active $\mathrm{C}_{60}$ molecules.

The absorption coefficient for the modulation beam is defined as

$$
\alpha\left(I_{m}\right)=\mathbf{N}\left(I_{m}\right) \cdot \boldsymbol{\sigma},
$$

where, the absorption cross-section vector $\sigma=\left(\sigma_{0}, \sigma_{S}, \sigma_{T}\right)$. Hence, using the population densities given in Eq. (3), the general expression for the absorption coefficient can be obtained from the above equation as $\alpha\left(I_{m}\right)=\alpha_{0}\left(1+A I_{m}\right)$, where, $\alpha_{0}=N_{1} \sigma_{0}$ and $A=\left(\tau_{S 0}^{-1}+\tau_{S T}^{-1}\right)^{-1}\left(\sigma_{S}+\sigma_{T} \tau_{T 0} \tau_{S T}^{-1}\right)$.

The feasibility of using $\mathrm{C}_{60}$ molecules for the development of a molecular SLM depends on the ability to modulate the weak probe signal by the intensity-dependent population densities in the excited states induced by the modulation laser beam. ${ }^{21-23}$ We analyze the modulation of the laser probe beams of intensity $I_{p}^{\prime}\left(\ll I_{m}^{\prime}\right)$ at two different wavelengths corresponding to the absorption maximum of singlet and the triplet states $S_{1}$ and $T_{1}$. The propagation of the probe beam through the $\mathrm{C}_{60}$ medium, in general, is governed by the equation

$$
\frac{d I_{p}}{d x}=-\alpha_{p} I_{p},
$$

where, $x$ is the distance in the medium and $\alpha_{p}$ is the absorption coefficient at the probe wavelength. Based on the absorption spectra of $S_{0}, S_{1}$, and $T_{1}$ states, ${ }^{17}$ the absorption coefficient for the respective probe beams can be written in terms of the modulation intensity-induced population densities, respectively, using Eqs. (3) and (4), as given in Table I. The modulation characteristics for the two probe beams can be computed by integrating Eq. (5) using the corresponding absorption coefficients. The results in the form of the ratio of the transmitted to the incident probe beam intensity are given in Table I.

The transmission characteristics of $\mathrm{C}_{60}$-toluene solution at two probe beams of wavelengths 747 and $885 \mathrm{~nm}$, corresponding to the absorption maximum of $T_{1}$ and $S_{1}$ states, as

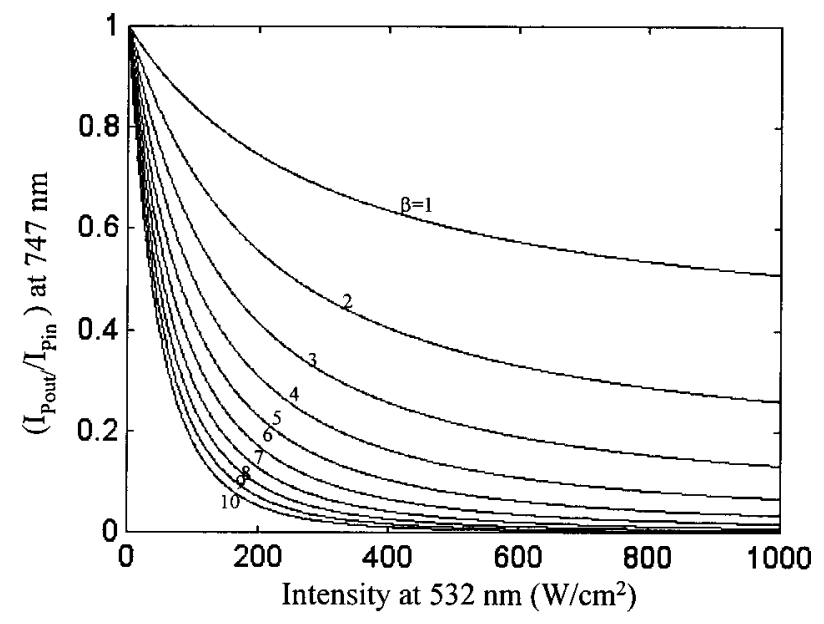

FIG. 2. Variation of the normalized intensity transmission of a probe beam at $747 \mathrm{~nm}$ with pump beam intensity at $532 \mathrm{~nm}$ for various values of the small-signal absorption coefficient.

a function of modulation light beam intensity $\left(I_{m}\right)$ at 532 $\mathrm{nm}$, are shown in Figs. 2 and 3, respectively, for different values of the small-signal absorption coefficient. The absorption cross sections and the relaxation times used in the computations are $\sigma_{0}=2.87 \times 10^{-18} \mathrm{~cm}^{2}, \sigma_{S}=1.57 \times 10^{-17} \mathrm{~cm}^{2}$, $\sigma_{T}=9.22 \times 10^{-18} \mathrm{~cm}^{2}, \quad \tau_{S 0}=30 \mathrm{~ns}, \tau_{T 0}=280 \mu \mathrm{s}$, and $\tau_{S T}$ $=1.2 \mathrm{~ns} .{ }^{17}$ Figures 2 and 3 indicate that the transmission of probe beams at both the wavelengths decreases considerably with increase in $I_{m}$ and attains a constant value for large values of $I_{m}$. The constant value for $747 \mathrm{~nm}$ transmission is reached for $I_{m}$ above $800 \mathrm{~W} / \mathrm{cm}^{2}$ while that for $885 \mathrm{~nm}$ is reached for intensities above $1000 \mathrm{~W} / \mathrm{cm}^{2}$. Figures 2 and 3 indicate that for high modulation, a $T_{1}$-based SLM requires much lower writing intensities. It is also seen from Figs. 2 and 3 that the modulation of the probe beam transmission is larger for higher values of small-signal absorption coefficient $\beta$. Hence, appreciable all-optical light modulation of the probe beam can be achieved by increasing the length of the active medium with higher concentration of $\mathrm{C}_{60}$ molecules.

This switching mechanism can be utilized for the construction of a molecular SLM based on $\mathrm{C}_{60}$ molecules. Such a SLM would be free from the problems encountered in conventional liquid-crystal or semiconductor SLMs due to the

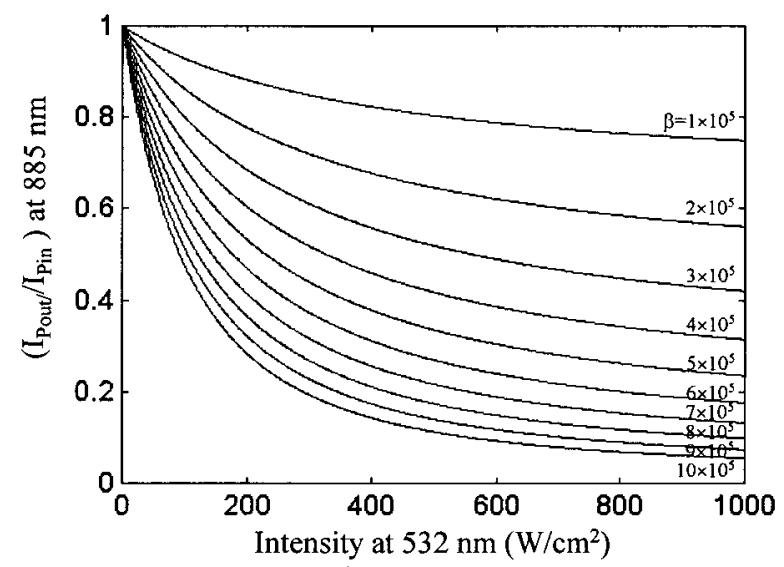

FIG. 3. Variation of the normalized intensity transmission of a probe beam at $885 \mathrm{~nm}$ with pump beam intensity at $532 \mathrm{~nm}$ for various values of the small-signal absorption coefficient. 
diffusion process, since the excitation is localized in the molecules. In this case, the resolution depends on the focusing geometry. Typical SLM properties can be estimated in the present case assuming the modulation beam as the write beam and the probe beam as the read beam. For example, the dynamic range, defined as the ratio of the transmitted intensity of the read beam through a sample of length $L$ in the presence of the write beam, to that in the absence of the write beam, can be calculated from the expression ${ }^{21,22}$

$$
\delta=\exp \left[\alpha_{R}(0) L-\alpha_{R}\left(I_{W}\right) L\right],
$$

where, $\alpha_{R}$ is the same as $\alpha_{p}$ and $I_{w}$ is the same as $I_{m}$ defined earlier. Since $\mathrm{C}_{60}$ has negligible absorption of the read beam by ground-state molecules for both singlet and triplet peak absorption wavelengths, $\alpha_{R}(0)=0$ for both 747 and $885 \mathrm{~nm}$ read beams, which corresponds to the ideal case. The variations in the dynamic range for both cases with write beam intensity is the same as the intensity modulation plotted in Figs. 2 and 3. Another important parameter of the SLM is the sensitivity, defined as ${ }^{21,22}$

$$
S=-\ln \delta / I_{W} .
$$

As a typical example, for the triplet state, for $\beta=4$, Fig. 2 yields $\delta=0.4$ and $S=9.16 \times 10^{-3} \mathrm{~cm}^{2} / \mathrm{W}$, for the write beam intensity of $100 \mathrm{~W} / \mathrm{cm}^{2}$ at $532 \mathrm{~nm}$, corresponding to $40 \%$ modulation. Thus, the SLM can be operated by a $5 \mu \mathrm{W}$ laser beam focused down to a $5 \mu \mathrm{m}^{2}$ spot size. The same modulation level can be achieved for the singlet state, for $\beta=7$ $\times 10^{5}$, as shown in Fig. 3. For $\beta=6$, for the $T_{1}$ state, Fig. 2 yields $\delta=0.9$ and $S=3.5 \times 10^{-4} \mathrm{~cm}^{2} / \mathrm{W}$ for the write beam intensity of $300 \mathrm{~W} / \mathrm{cm}^{2}$, corresponding to $90 \%$ modulation, which for the $S_{1}$ state, corresponds to $\beta=9 \times 10^{5}$ at 700 $\mathrm{W} / \mathrm{cm}^{2}$. For the $T_{1}$ state, $100 \%$ modulation can be achieved for $\beta=10$ at $800 \mathrm{~W} / \mathrm{cm}^{2}$, whereas, for the $S_{1}$ state, $94.5 \%$ modulation can be achieved for $\beta=10^{6}$ at $1 \mathrm{~kW} / \mathrm{cm}^{2}$.

SLMs are the important key elements in real-time optical signal processing, computing, and information processing systems. In the development of these devices, the current trend is to use man-made engineerable materials and structures that can be tailored for desired modulation characteristics in a particular application. Thus, the ability to tailor the structure and, hence, the properties of $\mathrm{C}_{60}$ by various techniques, ${ }^{14,15,19,20,24-26}$ makes it an attractive material for constructing a molecular SLM.
In conclusion, we have analyzed the transmission characteristics of $\mathrm{C}_{60}$-toluene solution using a simple rate equations approach. The analysis is applied to study the modulation of the transmission of probe beams at two different wavelengths by varying the intensity of the modulation beam at $532 \mathrm{~nm}$. It is found that appreciable modulation can be achieved by increasing the sample length and concentration of active molecules. The analysis demonstrates the feasibility of designing an optically addressed molecular spatial light modulator using $\mathrm{C}_{60}$ molecules.

${ }^{1}$ J. A. Neff, R. A. Athale, and S. H. Lee, Proc. IEEE 78, 826 (1990).

${ }^{2}$ K. P. J. Reddy, Curr. Sci. 61, 520 (1991).

${ }^{3}$ K. P. J. Reddy and P. K. Barhai, Pramana 35, 527 (1990).

${ }^{4}$ J. C. Ellenbogen and J. C. Love, Proc. IEEE 88, 386 (2000).

${ }^{5}$ F. T. S. Yu, Proc. IEEE 87, 1851 (1999).

${ }^{6}$ W. J. Blau and D. J. Cardin, Mod. Phys. Lett. B 6, 1351 (1992).

${ }^{7}$ H. S. Nalwa, Adv. Mater. 5, 341 (1993).

${ }^{8}$ L. W. Tutt and A. Kost, Nature (London) 356, 225 (1992).

${ }^{9}$ L. W. Tutt and T. F. Bogess, Prog. Quantum Electron. 17, 299 (1993).

${ }^{10}$ M. P. Joshi, S. R. Mishra, H. S. Rawat, S. C. Mehendale, and K. C. Rustagi, Appl. Phys. Lett. 62, 1763 (1993).

${ }^{11}$ J. E. Gray, K. C. Liu, C. H. Chen, W. R. Garrett, M. G. Payne, R. Goedert, and D. Templeton, Appl. Phys. Lett. 64, 2785 (1994).

${ }^{12}$ D. G. McLean, R. L. Sutherland, M. C. Brant, D. M. Brandelik, P. A. Fleitz, and T. Pottenger, Opt. Lett. 18, 858 (1993).

${ }^{13}$ F. Lin, J. Zhao, T. Luo, M. Jiang, Z. Wu, Y. Xie, Q. Qian, and H. Zeng, J. Appl. Phys. 74, 2140 (1993).

${ }^{14}$ Y. Song, G. Fang, Y. Wang, S. Liu, C. Li, L. Song, Y. Zhu, and Q. Hu, Appl. Phys. Lett. 74, 332 (1999).

${ }^{15}$ M. P. Joshi, J. Swiatkiewicz, F. Xu, P. N. Prasad, B. A. Reinhardt, and R. Kannan, Opt. Lett. 23, 1742 (1998).

${ }^{16}$ V. P. Belousov, I. M. Belousove, E. A. Gavrouskaya, V. A. Grigorev, O. B. Danilov, A. G. Kalintsev, V. E. Krasnopolskii, V. A. Smirnov, and E. N. Sosnov, Opt. Spectrosc. 87, 772 (1999).

${ }^{17}$ C. Li, L. Zhang, R. Wang, Y. Song, and Y. Wang, J. Opt. Soc. Am. B 11, 1356 (1994)

${ }^{18}$ F. Z. Henari, K. H. Cazzini, D. N. Weldon, and W. J. Blau, Appl. Phys. Lett. 68, 619 (1996)

${ }^{19}$ A. D. Xia, S. Wada, and H. Tashiro, Appl. Phys. Lett. 73, 1323 (1998).

${ }^{20}$ W. Gears, J. Poortmans, S. C. Jain, J. Nijs, R. Mertens, S. C. Veenstra, V. V. Krasnikov, and G. Hadziioannou, Sol. Energy Mater. Sol. Cells 61, 43 (2000).

${ }^{21}$ S. Speiser and M. Orenstein, Appl. Opt. 27, 2944 (1988).

${ }^{22}$ K. P. J. Reddy, J. Appl. Phys. 77, 6108 (1995).

${ }^{23}$ S. Roy and K. P. J. Reddy, Curr. Sci. 78, 184 (2000).

${ }^{24}$ T. Zhang, J. Li, P. Gao, Q. Gong, K. Tang, X. Jin, S. Zheng, and L. Li, Opt. Commun. 150, 210 (1998)

${ }^{25}$ J. Schell, D. Ohlmann, D. Brinkmann, R. Levy, M. Joucla, J. L. Rehspringer, and B. Honerlage, J. Chem. Phys. 111, 5929 (1999).

${ }^{26}$ Y. Song, C. Zhang, Y. Wang, G. Fang, C. Duan, S. Liu, X. Xin, and H. Ye, Opt. Commun. 168, 131 (1999). 